\title{
Análisis dimensional de la Escala de Dificultades en la Regulación Emocional (DERS-15) en universitarios mexicanos
}

\section{Dimensional Analysis of the Difficulties in Emotion Regulation Scale (DERS-15) in Mexican university students}

\author{
Anabel de la Rosa-Gómez * 1 , Alejandrina Hernández-Posadas ${ }^{1}$, \\ Pablo D. Valencia ${ }^{1}$, Daniel A. Guajardo-Garcini ${ }^{1}$ \\ 1 - Facultad de Estudios Superiores Iztacala, Universidad Nacional Autónoma de México. \\ Introducción \\ Método \\ Resultados \\ Discusión \\ Referencias
}

Recibido: 06/09/2020 Revisado: 08/12/2020 Aceptado: 09/01/2021

\section{Resumen}

La Escala de Dificultades en la Regulación Emocional (DERS) es un instrumento de gran interés en el campo de la investigación clínica y aplicada. Sin embargo, el estudio sobre sus propiedades psicométricas en la población mexicana ha sido limitado. El objetivo del estudio fue analizar la dimensionalidad de la DERS-15 en la población adulta mexicana a través de un diseño transversal, instrumental. Participaron 252 estudiantes universitarios del sistema a distancia, de entre 18 y 57 años $(\mathrm{M}=31.8, \mathrm{DE}=$ 8.9) seleccionados mediante un muestreo no probabilístico, intencional. Se realizó un análisis factorial confirmatorio, y con ello se probaron dos modelos factoriales (de dos y seis dimensiones). Un modelo de seis factores presentó un mejor ajuste. La consistencia interna y la confiabilidad de la escala completa resultaron adecuadas $\left(\omega_{\mathrm{H}}=.80\right)$.

Palabras clave: desregulación emocional, DERS, análisis factorial confirmatorio, adaptación, población mexicana

\begin{abstract}
The Difficulties in Emotion Regulation Scale (DERS) is an instrument of great interest in the fields of clinical and applied research. However, the study on its psychometric properties in Mexican population has been limited. The objective of this study was to analyse the dimensionality of DERS-15 in Mexican adult population, through an instrumental, cross-sectional design. 252 university students from the open and distance learning system participated, aged 18 to $57(\mathrm{M}=31.8, \mathrm{SD}=8.96)$, selected by non-probabilistic intentional sampling. A confirmatory factor analysis was performed, in which two factorial models were tested (two and six dimensions). A six-factor model demonstrated a better fit. The internal consistency and reliability of the entire scale were adequate $\left(\omega_{\mathrm{H}}=.80\right)$.
\end{abstract}

Keywords: emotional dysregulation, DERS, confirmatory factor analysis, adaptation, Mexican population

\footnotetext{
* Correspondencia a: Anabel de la Rosa Gómez, Av. De los Barrios Núm. 1, Los Reyes Iztacala, 54090 Tlalnepantla, Edo. de México. Torre de tutorías, $2^{\text {do } . ~ P i s o, ~ c u b i ́ c u l o ~} 22$. Teléfono: 52+(55) 56231344. E-mail: anabel.delarosa@iztacala.unam.mx

Cómo citar: De la Rosa-Gómez, A., Hernández-Posadas, A., Valencia, P. D., \& Guajardo-Garcini, D. (2021). Análisis dimensional de la Escala de Dificultades en la Regulación Emocional (DERS-15) en universitarios mexicanos. Revista Evaluar, 21(2), 80-97. Recuperado de https://revistas.unc.edu.ar/index.php/revaluar Participaron en la edición de este artículo: Tomás Milanesio, Pablo López Díaz, Alicia Molinari, Eugenia Barrionuevo, Florencia Ruiz, Benjamín Casanova, Ricardo Hernández.
} 


\section{Introducción}

En las últimas dos décadas se han logrado avances relevantes en el estudio de la regulación emocional. Específicamente, se ha logrado una comprensión más amplia de la trayectoria de su desarrollo, neuroanatomía, influencias genéticas y ambientales, y su vínculo con la cognición (Kring \& Sloan, 2010). La regulación emocional ha atraído el interés de los estudiosos del comportamiento en muchas disciplinas, y una de las razones es que ha incidido en las inquietudes tanto del ámbito científico como de la práctica clínica.

Las investigaciones en torno a la regulación emocional, entendida como el ejercicio de un control racional superior sobre los sistemas emocionales inferiores para lograr la adaptación, han aportado evidencia a la teoría de las emociones, incluyendo el rol fundamental que tiene la emoción en el funcionamiento adaptativo. Así, la regulación emocional puede ser estudiada desde diferentes aristas: desde los fundamentos neurobiológicos (Johnstone \& Walter, 2014), los procesos cognitivos de la experiencia emocional, y las influencias relacionales (Chervonsky \& Hunt, 2019), hasta las implicaciones culturales (Qu \& Telzer, 2017), los factores sociales y contextuales (de France \& Evans, 2020) y los aspectos temperamentales del individuo (van Beveren, de Clercq, \& Braet, 2020). Por lo tanto, su estudio implica oportunidades y retos para un análisis integral. El vínculo entre la regulación emocional y las estrategias de adaptación personal, la competencia social (Lebowitz \& Dovidio, 2015) e incluso el funcionamiento cognitivo, sugiere que la regulación emocional es un logro fundamental durante la etapa del desarrollo infantil y tiene consecuencias en la vida adulta. Esto ha contribuido a la conceptualización de muchas formas de psicopatología infantil y adulta (entre ellas la depresión, los trastornos de ansiedad, los proble- mas de conducta y otros trastornos internalizantes y externalizantes) como problemas de desregulación emocional. Debido a esto, nuevos enfoques terapéuticos se han desarrollado para mejorar las capacidades de autorregulación (Reyes-Ortega \& Tena-Suck, 2016).

Sin embargo, el estudio de la regulación emocional aborda una serie de desafíos conceptuales y empíricos. Por ejemplo, la identificación de estrategias de regulación emocional adaptativas y desadaptativas depende del contexto y el propósito (Aldao \& Nolen-Hoeksema, 2012), especialmente en condiciones de adversidad psicobiológica o ambiental; por lo tanto, no siempre se pueden desplegar estrategias adaptativas a largo plazo a pesar de que existan beneficios inmediatos. Además, el desarrollo de la regulación emocional es posible no solo por la maduración de funciones neurobiológicas, sino también por el desarrollo de una red compleja de procesos superiores involucrados. En este sentido, los investigadores continúan debatiendo su definición y características centrales (Campos, Frankel, \& Camras, 2004; Cole, Martin, \& Dennis, 2004; Gross \& Thompson, 2007; Thompson, 1994).

De este modo, de acuerdo con Gratz y Roemer (2004), la regulación emocional es la capacidad de comprender, aceptar y modular nuestros estados emocionales en busca de comportamientos dirigidos a objetivos. El uso efectivo de estrategias para la regulación emocional se ha asociado con un mejor bienestar psicológico y un mejor funcionamiento de las personas. Por el contrario, las dificultades para comprender, percibir y regular las emociones pueden contribuir a una amplia gama de resultados negativos (Berking \& Whitley, 2014; Yiğit \& Guzey-Yiğit, 2019).

La evidencia empírica ha demostrado que las dificultades en la regulación emocional son una característica esencial involucrada en el desarrollo y mantenimiento de múltiples formas de 
psicopatología (Bardeen, Kumpula, \& Orcutt, 2013; Tull, Bardeen, DiLillo, Messman-Moore, \& Gratz, 2015). Así, diversas manifestaciones de psicopatología se describen como excesos o déficit de la respuesta emocional; la experiencia abrumadora es más prevalente en muchos tipos de trastornos emocionales (Leahy, Tirch, \& Napolitano, 2011). En particular, la desregulación emocional del afecto negativo es un factor de estudio que está proporcionando datos relevantes para una mejor comprensión y abordaje de los trastornos psicológicos desde una perspectiva transdiagnóstica (Sauer-Zavala et al., 2017), incluyendo la depresión (Aldao \& Nolen-Hoeksema, 2010), el trastorno límite de la personalidad (Gratz \& Gunderson, 2006), la anorexia (Brockmeyer et al., 2012), el trastorno por consumo de sustancias (Wong et al., 2013) y el trastorno por estrés postraumático (Seligowski, Lee, Bardeen, \& Orcutt, 2015). Asimismo, se ha hallado relevante la función del despliegue de estrategias automáticas como los pensamientos negativos repetitivos y de estrategias más controladas como la reinterpretación o planificación en el modelo explicativo de la ideación suicida (Flores-Kanter, García-Batista, Moretti, \& Medrano, 2019).

Existe evidencia reciente que ha probado el efecto que tiene el estado de ánimo (valencia afectiva positiva y negativa) en el desarrollo de diversos juicios cognitivos tales como el autoconcepto, la autoeficacia, y juicios de desesperanza-optimismo (Flores-Kanter \& Medrano, 2020). A partir de estos hallazgos, se han desarrollado estudios que han logrado estudiar modelos explicativos más complejos que involucran (a) variables individuales (por ejemplo, sesgos de atención); (b) variables contextuales (por ejemplo, situaciones de estrés frente a situaciones de seguridad; estímulos emocionales positivos frente a negativos o estímulos neutrales frente a sobresalientes), y (c) las respuestas afectivo-cognitivas particulares que se desencadenan en cada caso y que impactan en la cognición (por ejemplo, en el momento de la elaboración del juicio; Flores-Kanter, 2020; Flores-Kanter \& Medrano, 2020).

Particularmente, a partir de los estudios de Linehan (1993) centrados en el trastorno límite de la personalidad es que se advirtió que la desregulación emocional resulta de dificultades para modular y tolerar las respuestas fisiológicas asociadas a las emociones. Por otro lado, Thompson (1994) definió a la regulación emocional como los procesos intrínsecos y extrínsecos involucrados en el monitoreo, evaluación y modulación de las reacciones emocionales con la finalidad del logro de objetivos, destacando como característica central la funcionalidad de la regulación para una situación particular.

Por su parte, Cole, Michel y Teti (1994) establecieron que la desregulación emocional no solo implica una falta de regulación, sino también un proceso regulatorio que opera de manera disfuncional. Se referían a una capacidad disminuida para suprimir estados afectivos negativos y el despliegue de respuestas emocionales inadecuadas para llevar a cabo actividades y comunicarse e influir en otros. Señalaron que es un proceso que tiene una función adaptativa en los individuos, incluso cuando interfiere en su desarrollo óptimo.

Como se señaló previamente, algunas conceptualizaciones se han centrado en la funcionalidad de las emociones dependiendo del contexto donde surgen y no solo en el control o inhibición de las emociones negativas (Cole et al., 1994; Thompson, 1994). Otras, se han enfocado en la importancia de aceptar y valorar las respuestas emocionales (Cole et al., 1994; Linehan, 1993), coincidiendo en que la tendencia a restringir y controlar la expresión emocional se asocia con un aumento de estimulación fisiológica (Gross \& Levenson, 1997). Asimismo, algunos autores enfatizan la importancia del contexto en el que 
se valoran las emociones, así como las demandas de la situación particular y las metas individuales (Cole et al., 1994; Thompson, 1994).

Dada la relevancia clínica de la regulación y desregulación emocional y la falta de un concepto preciso de este constructo, Gratz y Roemer (2004) propusieron una definición y medida integral de desregulación emocional. Para ellos, la desregulación emocional es un constructo multidimensional que involucra formas desadaptativas de responder a las emociones, incluyendo: (a) falta de conciencia y comprensión de las emociones, (b) falta de aceptación de las emociones, (c) incapacidad de controlar comportamientos impulsivos y comportarse de acuerdo a los objetivos deseados al experimentar emociones negativas, (d) incapacidad de utilizar estrategias de regulación emocional situacionalmente apropiadas y (e) flexibilidad para modular respuestas emocionales para cumplir con objetivos individuales y demandas situacionales.

Existen diversas escalas disponibles respaldadas empíricamente que permiten evaluar distintos procesos involucrados en la regulación emocional, por ejemplo, el Cuestionario de Regulación Emocional (ERQ; Gross \& John, 2003), el Cuestionario de Conciencia Emocional (Rieffe, Oosterveld, Miers, Meerum-Terwogt, \& Ly, 2008), el Cuestionario de Regulación Cognitivo Emocional (CERQ; Garnefski, Kraaij, \& Spinhoven, 2001) y la Medida de Estilos de Regulación Afectiva ampliada en Ira y Tristeza (MARS Ampliada; Páez-Rovira, MartínezSánchez, Sevillano-Triguero, Mendiburo-Seguel, \& Campos, 2012), entre otras.

Asimismo, Gratz y Roemer (2004) desarrollaron la Escala de Dificultades en Regulación Emocional (DERS, por sus siglas en inglés) para evaluar exhaustivamente las dificultades en la regulación emocional. Este instrumento es una medida de autoinforme de 36 ítems que comprende seis factores: (a) no aceptación de las respuestas emocionales (no-aceptación); (b) dificultades en conductas dirigidas a metas cuando se está alterado (metas); (c) dificultades para controlar comportamientos impulsivos cuando se está alterado (impulsividad); (d) acceso limitado a estrategias de regulación emocional percibidas como efectivas (estrategias); (e) falta de conciencia emocional (conciencia) y (f) falta de claridad emocional (claridad). La DERS ha demostrado adecuada robustez para su medición, adaptabilidad transcultural y evaluación de múltiples procesos implicados en la regulación emocional (Hallion, Steinman, Tolin, \& Diefenbach, 2018; Li, Han, Gao, Sun, \& Ahemaitijiang, 2018). Además, se han desarrollado varias versiones cortas para diversas poblaciones y contextos culturales que mostraron propiedades psicométricas adecuadas y pertinentes (por ejemplo, DERS-15; Muñoz-Martínez, Vargas, \& Hoyos-González, 2016; DERS-16, Bjureberg et al., 2016; Shahabi, Hasani, \& Bjureberg, 2020; DERS-SF, Kaufman et al., 2016, y DERS-18, Victor \& Klonsky, 2016).

\section{Las diferentes validaciones y propiedades psicométricas a nivel internacional}

La Escala de Dificultades en Regulación Emocional (DERS; Gratz \& Roemer, 2004) tiene su origen en el surgimiento de los modelos de la llamada tercera ola de la terapia cognitivo-conductual, que se basaron en las propuestas teóricas precedentes (Marín-Tejeda, Robles-García, González-Forteza, \& Andrade-Palos, 2012). El instrumento de medición fue diseñado para evaluar la capacidad de regulación emocional percibida a nivel de rasgo e identificar dificultades clínicamente relevantes (Medrano \& Trógolo, 2014); se ha citado en 3000 ocasiones y se ha traducido a varios idiomas (Hallion et al., 2018). Aunque es 
importante mencionar que a nivel mundial existe una gran diversidad de instrumentos que permiten evaluar la regulación emocional, para la etapa de la adultez se ha desarrollado una serie de instrumentos de evaluación que adopta, principalmente, la modalidad de entrevistas y cuestionarios (Guzmán-González, Trabucco, Urzúa, Garrido, \& Leiva, 2014). No obstante, no existe alguna escala que evalúe un abanico amplio de procesos como lo hace la escala DERS (Hervás \& Jódar, 2008). La DERS ha demostrado buenas propiedades psicométricas en muestras clínicas con diferentes edades (Muñoz-Martínez et al., 2016). Asimismo, Yiğit y Guzey-Yiğit (2019) han destacado la adaptabilidad intercultural del instrumento estableciendo así su validez transcultural y relevancia clínica.

En cuanto a la fiabilidad y validez, Gratz y Roemer, en 2004, informaron que la escala DERS original tiene una alta consistencia interna ( $\alpha=$ .93), una buena fiabilidad test-retest en un periodo de 4 a 8 semanas $(p=.88, p<.01)$ y una adecuada validez predictiva y de criterio (Hervás \& Jódar, 2008; Marín-Tejeda et al., 2012; Muñoz-Martínez et al., 2016). El análisis factorial exploratorio (AFE) sugirió una estructura de seis o siete factores (Hallion et al., 2018).

La DERS ha sido traducida, adaptada a diversos idiomas y validada en diferentes países, con resultados psicométricos variados (Medrano \& Trógolo, 2014; Muñoz-Martínez et al., 2016). En algunas situaciones replicó las características adecuadas de confiabilidad y validez de la escala original (Marín-Tejeda et al., 2012), como ha sido el caso de Estados Unidos (Weinberg \& Klonsky, 2009), Turquía (Ruganci \& Gençöz, 2010), Italia (Giromini, Velotti, de Campora, Bonalume, \& Cesare-Zavattini, 2012), España (Hervás \& Jódar, 2008), Argentina (Medrano \& Trógolo, 2014), Chile (Guzmán-González et al., 2014) y México (Marín-Tejeda et al., 2012). En cuanto a los casos de validación en Iberoamérica, para los que se ha utilizado la versión en castellano, en España el estudio realizado por Hervás y Jódar (2008) obtuvo una estructura factorial similar a la obtenida para la escala original en inglés, sin embargo, se encontró que algunos ítems se traslapan entre los factores de impulsividad y estrategias (Guzmán-González et al., 2014; Hervás \& Jódar, 2008; Muñoz-Martínez et al., 2016). En el caso de México, se cuenta con la investigación con población adolescente realizada por Marín-Tejeda et al. (2012), donde los estudios de análisis factorial exploratorio y confirmatorio indicaron un modelo de cuatro factores. En esta versión, los 24 ítems obtenidos se agruparon en las siguientes dimensiones: no aceptación de las respuestas emocionales, dificultades para dirigir el comportamiento hacia metas, falta de conciencia emocional y falta de claridad emocional.

Medrano y Trógolo (2014) realizaron en Argentina un AFE con rotación promax y una estimación ponderada de mínimos cuadrados para establecer la estructura factorial de la escala. La versión argentina de la DERS tiene 28 ítems agrupados en seis factores, que explicaban el 50.79\% de la varianza (Medrano \& Trógolo, 2014).

En Brasil se realizó la validación del instrumento con una muestra comunitaria adulta, y se obtuvieron buenas propiedades psicométricas, con evidencias de validez y confiabilidad, confirmando 6 factores con 36 ítems (Mattos-Machado, Gonçalves-Gurgel, Gonçalves-Boeckel, \& TozziReppold, 2020). En tanto, en Chile, GuzmánGonzález et al. (2014) probaron las propiedades psicométricas de la DERS-E en estudiantes universitarios y en la población general chilena, y se discriminaron cinco factores, incluyendo un total de 25 ítems.

En Colombia, Herrera, Niño, Caycedo y Cortés (2008, citado en Muñoz-Martínez et al., 2016) realizaron el análisis de confiabilidad para 
la DERS y obtuvieron un alto índice de confiabilidad $(\alpha=.90)$ en una muestra de estudiantes bogotanos. Por su parte, Muñoz-Martínez et al. (2016) buscaron identificar la consistencia de los factores incluidos en la DERS y la relevancia de los ítems en cada dimensión estudiada empleando la versión de 36 ítems compuesta por (a) no-aceptación, (b) metas, (c) impulsividad, (d) estrategias, (e) consistencia y (f) claridad. Mediante análisis factorial exploratorio se obtuvieron dos factores principales en lugar de seis, y se realizó una reducción en el número de reactivos, conservando 15 de los 36 ítems originales. Puesto que el segundo factor se integra únicamente por un ítem, los autores advirtieron que se mantuvieron dos factores debido a que contribuían significativamente a la varianza: el Factor 1 (integrado por los subdimensiones no-aceptación, metas, impulsividad, estrategias y claridad) y el Factor 2 (integrado por la dimensión conciencia) mostraron independencia. En específico, la independencia del factor conciencia con respecto al otro factor se fundamenta en las bases teóricas de la regulación emocional (Gratz \& Roemer, 2004), que la consideran relacionada con la forma de entender y atender las emociones. No obstante, existen otras conceptualizaciones que la señalan como un repertorio amplio de estrategias a partir de las cuales las personas se vinculan con sus reacciones emocionales (Stewart, Villate, \& McHugh, 2012), por lo que podría incluir las relaciones de cualquier tipo de comportamiento con el ambiente que lo influye. Así, la conciencia implica darse cuenta de las propias acciones sin alterarlas, regularlas o modularlas (Muñoz-Martínez et al., 2016). Así, existe controversia sobre si la conciencia podría ser un factor independiente del constructo de regulación o desregulación emocional, aunque algunos autores sugieren que podría ser una estrategia para la RE (Ruganci \& Gençöz, 2010; Vargas-Gutiérrez \& Muñoz-Martínez,
2013; Weiss, Gratz, \& Lavender, 2015).

Es así que la escala DERS parece ser un instrumento de interés tanto en el campo de la investigación clínica como en el ámbito aplicado (Hervás \& Jódar, 2008). No obstante, la investigación sobre sus propiedades psicométricas en la población mexicana ha sido limitada. Se cuenta, por ejemplo, con un estudio realizado en adolescentes mexicanos (Marín-Tejeda et al., 2012); sin embargo, no se han encontrado estudios de validación en población adulta. Ante ello urge explorar alternativas breves ya que, como mencionan Flores-Kanter y Medrano (2018), el uso de escalas breves o reducidas (short-form) facilita las respuestas cuando hay muchas variables siendo medidas, o cuando, por el contexto, hay un tiempo limitado para responder la versión completa del instrumento. En este sentido, la DERS-15 (Muñoz-Martínez et al., 2016) presentó propiedades psicométricas adecuadas en su versión breve en español, lo cual facilitará la evaluación pertinente y eficiente de las dificultades en regulación emocional que presenta la población adulta mexicana, y con ello, permitirá potenciar la eficacia de las intervenciones preventivas o remediales.

De esta manera, el objetivo del presente trabajo fue analizar la dimensionalidad de la DERS-15 en la población mexicana adulta. Para ello, se probaron dos modelos factoriales (uno de dos y otro de seis dimensiones). Adicionalmente se examinó si, en el modelo de seis dimensiones, las cinco dimensiones restantes luego de excluir conciencia podrían ser englobadas en una única dimensión. Finalmente, se estimó la confiabilidad de esta dimensión global.

\section{Método \\ Participantes}

En el estudio participaron 252 estudiantes 
del primer semestre de la licenciatura de psicología del Sistema de Universidad Abierta y Educación a Distancia (SUAyED) del ciclo escolar 2018-2. Sus edades se hallaron entre los 18 y 57 años con una media de $31.8(\mathrm{DE}=8.96)$. La mayoría fueron mujeres. En la Tabla 1 se presenta la información sociodemográfica detallada de los participantes.

\section{Tabla 1}

Datos sociodemográficos de los participantes.

\begin{tabular}{lcr}
\hline Variable & $\mathbf{n}$ & $\mathbf{\%}$ \\
\hline Sexo & & \\
Mujer & 178 & 70.63 \\
Hombre & 74 & 29.30
\end{tabular}

Estado civil

Soltero

Unión libre

Casado

Viudo

16

Hijos

No

Sí

Lugar de residencia

\begin{tabular}{lcr} 
Ciudad de México & 105 & 41.67 \\
Estado de México & 55 & 21.83 \\
Oaxaca & 19 & 7.54 \\
Tlaxcala & 11 & 4.37 \\
Puebla & 11 & 4.37 \\
Otro & 51 & 20.24 \\
& & \\
Ocupación & & \\
Trabajo remunerado & 166 & 65.87 \\
Solo estudios & 68 & 26.98 \\
Trabajo y estudios & 18 & 7.14 \\
\hline
\end{tabular}

El muestreo se realizó de forma no probabilística, intencional, a través de la técnica de encuesta. Así, se invitó a participar a los estudiantes a través de medios de comunicación institucional (plataforma educativa, redes sociales institucionales). Todos los participantes aceptaron el consentimiento informado para la evaluación voluntaria y fueron notificados sobre el aviso de privacidad y confidencialidad de datos. La aplicación se realizó a distancia a través de un sistema de encuestas en línea. Como criterios de inclusión, se estableció que los participantes debían ser: 1) mayores de edad; 2) estudiantes de la licenciatura en Psicología del SUAyED, y 3) de nacionalidad mexicana.

\section{Instrumento}

Escala de Dificultades en Regulación Emocional (DERS). El instrumento original es una escala de autoinforme que cuenta con 36 ítems, los cuales se dividen en 6 factores: 1) no aceptación, 2) metas, 3) impulsividad, 4) estrategias, 5) conciencia y 6) claridad. Las autoras de la versión original informaron un coeficiente alfa de .88 para toda la escala (Gratz \& Roemer, 2004). En el presente estudio se empleó la versión breve del instrumento validada en Colombia, la cual cuenta con 15 ítems agrupados en dos factores (DERS-15; Muñoz-Martínez et al., 2016). El primer factor se compone por ítems que originalmente pertenecían a las dimensiones de no aceptación, metas, impulsividad, estrategias y claridad. Por otra parte, el segundo factor solo incluye un ítem que pertenecía a la dimensión conciencia. Esta versión de la DERS mostró un alto índice de confiabilidad $(\alpha=.90)$ en el estudio de Muñoz-Martínez et al. (2016). 
Diseño

El diseño del presente estudio fue transversal, pues los datos se obtuvieron en un solo momento. Asimismo, fue instrumental, pues tuvo como finalidad examinar las propiedades psicométricas de un instrumento (Montero \& León, 2007).

\section{Procedimiento}

Se empleó el instrumento DERS-15 validado en población adulta colombiana (MuñozMartínez et al., 2016). Un grupo de jueces expertos $(\mathrm{N}=10)$ evaluaron todos los ítems que componen la DERS-15; los expertos contaban con grado académico de doctorado en psicología y experiencia en el área clínica y de la salud. La validación por jueces consistió en conocer si cada ítem cumplía o no con los criterios de pertinencia, lenguaje, redacción y validez teórica. A fin de determinar la validez de contenido de la DERS-15 se realizó el cálculo del Índice de Acuerdo entre Jueces (IA) y el Coeficiente V de Aiken (V), considerando que una buena validez de contenido comprende un valor igual o superior al $80 \%$ (V $=.80)$ de acuerdo entre los jueces evaluadores (Kerlinger \& Lee, 2002). Se identificó que la mayoría de los ítems estimaba un índice de acuerdo entre jueces superior al $80 \%$ y un coeficiente $\mathrm{V}$ de Aiken superior a .80 para todos los criterios de evaluación establecidos. Los ítems que obtienen coeficientes superiores al $80 \%(\mathrm{~V}=.80)$ estiman un buen grado de dominio teórico sobre el elemento que pretende medir, mientras que los ítems que están por debajo de este valor requieren ser eliminados o sometidos a la realización de las modificaciones pertinentes. Pese a que la mayoría de los ítems estimó un buen grado de dominio teórico, se identificó que la redacción y el lenguaje de dos ítems no eran apropiados para el contexto mexicano: a) ítem 9, Yo me siento confundido acerca de cómo me siento, y b) ítem 14, Cuando estoy molesto, quedo fuera de control. Por lo tanto, se modificaron con base en las sugerencias de los expertos, y quedaron formulados del siguiente modo: a) ítem 9, Estoy confundido acerca de cómo me siento, y b) ítem 14, Cuando estoy molesto, me siento fuera de control.

La aplicación del instrumento se realizó a distancia a través de un sistema de encuesta en línea. Se envió una invitación para participar de manera voluntaria y se compartió el enlace del instrumento con la finalidad de conocer la regulación emocional de los participantes en diversas situaciones de la vida cotidiana. Los interesados brindaron su consentimiento informado para responder la escala y se enfatizó el trato confidencial de la información y el uso protegido de los datos personales. El cuestionario en línea estuvo habilitado durante un mes, y semanalmente se enviaron notificaciones reiterando la invitación a participar; a aquellos que expresaron su rechazo a participar se les dejó de invitar. Finalmente, al término del mes se cerró el formulario y se enviaron informes personales con los resultados de la escala y un catálogo de servicios psicológicos en modalidad a distancia y presencial atendiendo al caso de que el participante deseara atender su malestar emocional. La recolección de datos se realizó durante el año 2018.

\section{Análisis de datos}

Se examinó, en primer lugar, la media, la desviación estándar, la asimetría y la curtosis de cada uno de los ítems. Debido a que el instrumento cuenta con al menos cinco opciones de respuesta, fue posible tratar cada uno de los indicadores como si fuesen variables cuantitativas (Rhemtulla, 
Brosseau-Liard, \& Savalei, 2012). Por ello, se procedió a realizar un análisis factorial confirmatorio (AFC) basado en correlaciones de Pearson y utilizando una variante robusta del método de máxima verosimilitud, la cual permite corregir la falta de normalidad multivariada (MLR; Yuan \& Bentler, 2000). El ajuste de cada modelo se evaluó a partir de los siguientes índices (se menciona entre paréntesis el criterio de buen ajuste): CFI $(>.95)$, TLI $(>.95)$, RMSEA $(<.06)$ y SRMR $(<$ .08; Hu \& Bentler, 1999). Para el CFI, el TLI y el RMSEA, se usaron fórmulas modificadas que son consideradas más adecuadas cuando se utilizan estimadores robustos (Brosseau-Liard, Savalei, \& Li, 2012; Brosseau-Liard \& Savalei, 2014). Estas modificaciones consisten en incluir el factor de escalamiento en las fórmulas de los índices, de manera tal que estos puedan ser interpretados como los valores que se habrían obtenido si los datos siguieran una distribución normal.

El AFC puso a prueba dos modelos competidores. El primer modelo fue el propuesto en la adaptación colombiana, el cual agrupa todos los ítems en un factor denominado estrategias, a excepción del único ítem de la dimensión conciencia, que es separado en un factor distinto (Muñoz-Martínez et al., 2016). El segundo estuvo basado en el modelo original de seis factores (Gratz \& Roemer, 2004). En ambos modelos se incluyeron factores que contenían un único indicador, lo cual constituyó una limitación para la forma tradicional de realizar este tipo de análisis (Byrne, 2012). Una posible solución a este problema sería incluir el ítem único como variable observada en el modelo o, de manera equivalente, como un único indicador perfecto de la variable latente correspondiente. Sin embargo, en ambos casos se asumiría que la variable es medida sin error, lo cual es poco realista al medir atributos psicológicos. Por ello, un compromiso intermedio es establecer arbitrariamente un valor de error, para lo cual el investigador debe asumir la confiabilidad $(\rho)$ del indicador. Dicho término de error será igual a s${ }^{2}{ }_{\mathrm{Yi}}(1-\rho)$, donde $\mathrm{s}^{2}$ Yi es la varianza observada del indicador (Brown, 2015). En el presente estudio se decidió establecer un valor de $\rho=.80$, siguiendo las recomendaciones de estudios de simulación existentes (Savalei, 2019). Cabe precisar que el modelado de variables latentes con un único indicador es un aspecto discutido ampliamente en la bibliografía metodológica (Petrescu, 2013) y ha sido aplicado anteriormente al campo de la psicometría (Caycho-Rodríguez, Dominguez-Lara, Noe-Grijalva, \& Reyes-Bossio, 2019; Dominguez-Lara, 2018).

Luego de poner a prueba los dos modelos confirmatorios descritos, se examinó la unidimensionalidad esencial de la escala en su totalidad (a excepción del ítem de la dimensión conciencia). Para ello, se realizó un análisis exploratorio bifactor (no fue posible realizarlo con un AFC debido a que el factor específico no aceptación tenía un solo indicador). Este modelo bifactor se estimó a partir de una matriz diana y una rotación procrusteana ortogonal (Browne, 1972). La unidimensionalidad esencial de los 14 ítems se juzgó a partir de la varianza común explicada (ECV) por el factor general (Flores-Kanter, Dominguez-Lara, Trólogo, \& Medrano, 2018). Un valor de ECV mayor a .85 indicaría que la escala es esencialmente unidimensional y que, por lo tanto, un puntaje total es más robusto que los puntajes por dimensiones (Rodriguez, Reise, \& Haviland, 2016).

Luego de justificar el cálculo de puntajes separados para cada dimensión, se usó el modelo confirmatorio de seis factores para estimar la confiabilidad de cada dimensión. Para ello, se utilizó el coeficiente omega, el cual presenta ventajas en comparación con el tradicionalmente usado coeficiente alfa de Cronbach (Dunn, Baguley, \& Brunsden, 2014).

El análisis factorial confirmatorio se llevó a 
Tabla 2

Estadísticos descriptivos de los ítems de la DERS-15.

\begin{tabular}{|c|c|c|c|c|c|}
\hline Ítem & $\begin{array}{c}\text { Dimensión } \\
\text { original }\end{array}$ & Media & $\mathbf{D E}$ & Asimetría & Curtosis \\
\hline $\begin{array}{l}\text { 1. Tengo dificultad para encontrar el significado a mis } \\
\text { sentimientos. }\end{array}$ & Claridad & 2.03 & 1.08 & 1.13 & 0.65 \\
\hline 2. Yo me siento confundido acerca de cómo me siento. & Claridad & 1.97 & 1.07 & 1.18 & 0.77 \\
\hline $\begin{array}{l}\text { 3. Cuando estoy alterado, tengo dificultad para realizar el } \\
\text { trabajo. }\end{array}$ & Metas & 2.50 & 1.24 & 0.70 & -0.61 \\
\hline 4. Cuando estoy molesto, quedo fuera de control. & Impulsividad & 1.77 & 1.01 & 1.34 & 1.11 \\
\hline $\begin{array}{l}\text { 5. Cuando estoy alterado, creo que seguirá siendo así } \\
\text { durante mucho tiempo. }\end{array}$ & Estrategias & 1.69 & 1.05 & 1.68 & 2.11 \\
\hline $\begin{array}{l}\text { 6. Cuando estoy alterado, creo que voy a terminar } \\
\text { sintiéndome muy deprimido. }\end{array}$ & Estrategias & 1.80 & 1.17 & 1.53 & 1.36 \\
\hline $\begin{array}{l}\text { 7. Cuando estoy alterado, tengo dificultad para } \\
\text { concentrarme en otras cosas. }\end{array}$ & Metas & 2.67 & 1.31 & 0.51 & -1.01 \\
\hline 8. Cuando estoy alterado, me siento fuera de control. & Impulsividad & 1.88 & 1.15 & 1.31 & 0.77 \\
\hline $\begin{array}{l}\text { 9. Cuando estoy alterado, me siento avergonzado de mí } \\
\text { mismo por sentir de esa manera. }\end{array}$ & $\begin{array}{l}\text { No acepta- } \\
\text { ción }\end{array}$ & 1.85 & 1.14 & 1.36 & 0.92 \\
\hline $\begin{array}{l}\text { 10. Cuando estoy alterado, yo tengo dificultades } \\
\text { concentrándome. }\end{array}$ & Metas & 2.68 & 1.28 & 0.54 & -0.93 \\
\hline $\begin{array}{l}\text { 11. Cuando estoy alterado, tengo dificultades controlando } \\
\text { mis comportamientos. }\end{array}$ & Impulsividad & 1.99 & 1.13 & 1.10 & 0.40 \\
\hline $\begin{array}{l}\text { 12. Cuando estoy alterado, creo que no hay nada que } \\
\text { pueda hacer para sentirme mejor. }\end{array}$ & Estrategias & 1.84 & 1.14 & 1.37 & 0.88 \\
\hline $\begin{array}{l}\text { 13. Cuando estoy alterado, pierdo el control sobre mis } \\
\text { conductas. }\end{array}$ & Impulsividad & 1.65 & 1.02 & 1.80 & 2.72 \\
\hline $\begin{array}{l}\text { 14. Cuando estoy alterado, encuentro difícil pensar en } \\
\text { algo más. }\end{array}$ & Metas & 2.29 & 1.21 & 0.88 & -0.19 \\
\hline 15. Yo estoy atento a mis sentimientos. (r) & Conciencia & 2.46 & 1.21 & 0.42 & -0.95 \\
\hline
\end{tabular}

Nota. $(r)=$ Ítem de calificación invertida.

cabo en el programa lavaan 0.6-7 (Rosseel, 2012). Por su parte, el análisis de unidimensionalidad esencial con un modelo bifactor se realizó con FACTOR 10.10.02 (Ferrando \& Lorenzo-Seva, 2017). Finalmente, para el cálculo del coeficiente omega se utilizó el paquete semTools 0.5-3. Tanto lavaan como semTools fueron ejecutados en R 4.0.3.

\section{Resultados \\ Estadísticos descriptivos}

Los estadísticos descriptivos al nivel de los ítems son presentados en la Tabla 2. Como se observa, existió una notable variabilidad en cuanto a las medias de los ítems, las cuales fluctuaron entre 1.65 (ítem 13) y 2.67 (ítem 7). Algo menor 
Tabla 3

Índices de ajuste de los modelos analizados.

\begin{tabular}{lcccccc}
\hline Modelo & MLR $\boldsymbol{\chi}^{2}$ & $\boldsymbol{g l}$ & CFI & TLI & RMSEA & SRMR \\
\hline 2 factores & 493.90 & 90 & .77 & .74 & .16 & .09 \\
6 factores & 162.87 & 77 & .95 & .94 & .08 & .04 \\
\hline
\end{tabular}

Tabla 4

Correlaciones latentes del modelo de 6 factores de la DERS- 15 .

\begin{tabular}{lcccccc}
\hline Dimensión & $\mathbf{1}$ & $\mathbf{2}$ & $\mathbf{3}$ & $\mathbf{4}$ & $\mathbf{5}$ & $\mathbf{6}$ \\
\hline 1. Estrategias & 1 & & & & & \\
2. Impulsividad & .91 & 1 & & & & \\
3. Metas & .80 & .69 & 1 & 1 & 1 & .06 \\
4. Claridad & .63 & .48 & .48 & .48 & .44 \\
5. No aceptación & .63 & .63 & .52 & .03 & & \\
6. Conciencia & .23 & .13 & & & & \\
\hline
\end{tabular}

fue la variabilidad existente entre las desviaciones estándar, la mayoría de las cuales se halló entre 1 y 1.2. Finalmente, se aprecian valores elevados de asimetría y curtosis en algunos ítems, especialmente en los ítems 5 y 13.

\section{Análisis factorial confirmatorio}

Para analizar el modelo de dos factores, se igualó el error del ítem 15 (indicador único de conciencia) al valor $s_{Y 15}^{2}(1-\rho)=1.21^{2}(1-.8)=$ .29. Como se observa en la Tabla 3 , el ajuste de este modelo fue mediocre según todos los índices examinados. Por otra parte, la correlación interfactorial fue baja $(\varphi=.163)$.

Asimismo, al probar el modelo de seis factores, se ajustó el ítem 9 a tener un error igual a $S_{Y 9}^{2}(1-\rho)=1.14^{2}(1-.8)=.26$, además del ítem 15 ya mencionado en el párrafo anterior. Como se observa en la Tabla 3, este modelo mostró un mejor ajuste en la mayoría de los índices apro- ximados (a excepción del RMSEA y, marginalmente, del TLI). Se examinaron las correlaciones interfactoriales y se evidenció que, salvo el factor conciencia, el resto de dimensiones mostraron elevadas correlaciones entre sí (Tabla 4). A partir de este resultado, se procedió a probar si estas dimensiones podrían, en realidad, ser capturadas por una única dimensión global.

\section{Análisis de la unidimensionalidad esencial}

Se examinaron los ítems de estrategias, impulsividad, metas, claridad y no aceptación a través de un modelo bifactor exploratorio. Las cargas factoriales de este modelo, así como las de los dos modelos confirmatorios presentados anteriormente, se muestran en la Tabla 5. La ECV calculada a partir de los resultados del modelo bifactor fue de .57 , lo cual no apoya la hipótesis de unidimensionalidad esencial. 
Estimación de la confiabilidad

Debido a que la hipótesis de unidimensionalidad esencial no fue apoyada por el análisis bifactor, se decidió mantener como modelo final de la DERS-15 aquel compuesto por las seis dimensiones originales. Fue posible estimar la confiabilidad de cuatro de estas: estrategias $(\omega=$ $.84)$, impulsividad, $(\omega=.91)$, metas $(\omega=.91) \mathrm{y}$ claridad $(\omega=.83)$. En cuanto a las dos dimensiones restantes (no aceptación y conciencia), dicha estimación no fue posible, pues ambas resultaron variables latentes con un solo indicador.

\section{Discusión}

Considerando que uno de los principales desafíos a la perspectiva caracterológica se deriva de la evidencia de síntomas extensivos y de comorbilidad tanto en la ansiedad como en la depresión, un amplio grupo de investigaciones epidemiológicas, diagnósticas y basadas en síntomas ha desafiado este enfoque categorial de la nosología psiquiátrica, ofreciendo evidencias mucho más firmes sobre la naturaleza dimensional de los trastornos psiquiátricos como la ansiedad o la depresión (Barlow, Allen, \& Choate, 2004; Kerlinger \& Howard, 2002). Sin embargo, las discusiones actuales entre los cuerpos colegiados permiten vislumbrar que los instrumentos de medición en los próximos años seguirán siendo los autoinformes (Hernández-Guzmán, del Palacio, Freyre, \& Alcázar-Olán, 2011).

Ante la demanda creciente en nuestro país para realizar diagnóstico y tratamiento de los trastornos emocionales, en particular de medir factores asociados tales como la regulación y la desregulación emocional, el presente estudio examinó las propiedades psicométricas de la Escala de Dificultades en Regulación Emocional
(DERS-15; Muñoz-Martínez et al., 2016) adaptada para estudiantes universitarios mexicanos. Este estudio se realizó debido a que no se contaba con una versión válida y confiable para este grupo etario y, asimismo, para evitar el error metodológico de utilizar traducciones de instrumentos desarrolladas para otras culturas (American Psychiatric Association, 2013), como es el caso de este cuestionario que continúa vigente y que posee buenas características psicométricas con población anglosajona y latinoamericana, pero que a la fecha no contaba con una versión validada para adultos mexicanos.

Los resultados mostraron que un modelo de seis factores basado en el original presentaba un mejor ajuste que un modelo de dos factores. A excepción del factor conciencia, todas las otras dimensiones mostraron correlaciones entre moderadas y altas. Sin embargo, a partir del modelado bifactor, no se halló evidencia de que estas dimensiones pudieran ser combinadas en una sola. Finalmente, se estimó la confiabilidad por consistencia interna y se encontraron valores elevados.

Se señala que los ítems de la DERS-15 en esta muestra estudiantil mexicana se distribuyeron en seis factores en concordancia con el modelo original de Gratz y Roemer (2004), así como con los hallazgos de otros estudios (Giromini et al., 2012; Medrano \& Trógolo, 2014). Por otra parte, estos resultados no concuerdan con lo informado por Muñoz-Martínez et al. (2016), quienes, al realizar el análisis factorial exploratorio, hallaron que los ítems de la DERS se reunieron en dos factores principales en lugar de seis.

Estos resultados pueden deberse a diferencias sociodemográficas y, en particular, a la edad de las muestras estudiadas; en las poblaciones latinoamericanas se aplicaron a jóvenes universitarios, mientras que en el presente estudio se evaluó a universitarios mexicanos de la modalidad a distancia, cuyo rango de edad fue de entre 18 y 57 
Tabla 5

Cargas factoriales de los modelos probados para la DERS-15.

\begin{tabular}{|c|c|c|c|c|c|c|c|c|c|c|c|c|c|c|}
\hline \multirow[t]{2}{*}{ Ítem } & \multicolumn{2}{|c|}{ Modelo de 2 factores } & \multicolumn{6}{|c|}{ Modelo de 6 factores } & \multicolumn{6}{|c|}{ Modelo bifactor (exploratorio) } \\
\hline & DES & CON & EST & IMP & MET & CLA & NOA & CON & EST & IMP & MET & CLA & NOA & DES \\
\hline 1 & .48 & & & & & .84 & & & .55 & .03 & -.04 & .12 & .00 & .51 \\
\hline 2 & .51 & & & & & .84 & & & .70 & -.09 & -.08 & -.01 & -.03 & .63 \\
\hline 3 & .70 & & & & .79 & & & & .07 & .52 & .08 & .15 & .00 & .58 \\
\hline 4 & .80 & & & .85 & & & & & .04 & .16 & .59 & .21 & .01 & .60 \\
\hline 5 & .80 & & .83 & & & & & & .09 & .15 & .36 & .43 & .00 & .62 \\
\hline 6 & .76 & & .78 & & & & & & .13 & .18 & .12 & .32 & .05 & .68 \\
\hline 7 & .73 & & & & .90 & & & & -.03 & .63 & .02 & .11 & .01 & .63 \\
\hline 8 & .81 & & & .87 & & & & & .00 & .18 & .59 & .24 & .14 & .60 \\
\hline 9 & .58 & & & & & & .89 & & -.02 & -.06 & .04 & -.02 & .74 & .67 \\
\hline 10 & .75 & & & & .91 & & & & .02 & .66 & .06 & -.09 & .03 & .68 \\
\hline 11 & .81 & & & .86 & & & & & -.11 & -.01 & .38 & .11 & -.02 & .77 \\
\hline 12 & .78 & & .79 & & & & & & -.11 & .05 & .06 & .32 & -.08 & .78 \\
\hline 13 & .75 & & & .82 & & & & & -.06 & -.13 & .48 & -.02 & -.09 & .74 \\
\hline 14 & .78 & & & & .80 & & & & -.12 & .35 & .03 & .22 & -.10 & .73 \\
\hline 15 & & .89 & & & & & & .89 & - & - & - & - & - & - \\
\hline
\end{tabular}

Nota. $\mathrm{DES}=$ desregulación, $\mathrm{CON}=$ conciencia, $\mathrm{EST}=$ estrategias, IMP $=$ impulsividad, $\mathrm{MET}=$ metas, $\mathrm{CLA}=$ claridad, NOA = no aceptación. 
años, en su mayoría mujeres que combinan sus estudios con el trabajo y que están al cuidado de sus hijos. Por lo tanto, la naturaleza y el tipo de representatividad de la muestra deben ser consideradas, además de las diferencias culturales.

Entre las limitaciones del estudio se encuentran el tamaño y la distribución de la muestra, que impide la generalización de los resultados a toda la población. Por ello se recomienda llevar a cabo otras investigaciones en muestras más amplias para evaluar el desempeño de la DERS con muestras distribuidas normalmente, así como indagar la utilidad y sensibilidad de la escala, tanto en población clínica como no clínica, para detectar y predecir distintas psicopatologías asociadas a la desregulación emocional, con su consecuente beneficio para el tratamiento de las mismas. Estudios futuros también deberán examinar otros modelos, como el de Guzmán-González et al. (2014), quienes hallaron un modelo de cinco factores en población chilena, y el de Marín-Tejeda et al. (2012) quienes identificaron un nuevo modelo de cuatro factores en adolescentes mexicanos. Asimismo, consideramos conveniente, en futuros trabajos, estimar la validez de criterio mediante el análisis de la validez convergente y discriminante de la DERS en población mexicana.

\section{Referencias}

American Psychiatric Association. (2013). DSM-5 Overview: The Future Manual. DSM-5 development. Recuperado de http://www.dsm5.org/about/Pages/ DSMVOverview.aspx

Aldao, A., \& Nolen-Hoeksema, S. (2010). Specificity of cognitive emotion regulation strategies: A transdiagnostic examination. Behaviour Research and Therapy, 48(10), 974-983. doi: 10.1016/j.brat.2010.06.002

Aldao, A., \& Nolen-Hoeksema, S. (2012). The influence of context on the implementation of adaptive emotion regulation strategies. Behaviour Research and Therapy, 50(7-8), 493-501. doi: 10.1016/j. brat.2012.04.004

Barlow, D. H., Allen, L. B., \& Choate, M. L. (2004). Toward a unified treatment for emotional disorders. Behavior Therapy, 35(2), 205-230. doi: 10.1016/ S0005-7894(04)80036-4

Bardeen, J. R., Kumpula, M. J., \& Orcutt, H. K. (2013). Emotion regulation difficulties as a prospective predictor of posttraumatic stress symptoms following a mass shooting. Journal of Anxiety Disorders, 27(2), 188-196. doi: 10.1016/j.janxdis.2013.01.003

Berking, M., \& Whitley, B. (2014). Emotion regulation: Definition and relevance for mental health. En M. Berking \& B. Whitley (Eds.), Affect regulation training: A practitioners' manual (pp. 5-17). New York, NY: Springer. doi: 10.1007/978-1-4939-1022-9_2

Bjureberg, J., Ljótsson, B., Tull, M. T., Hedman, E., Sahlin, H., Lundh, L. G., ... \& Gratz, K. L. (2016). Development and validation of a brief version of the difficulties in emotion regulation scale: The DERS-16. Journal of Psychopathology Behaviour Assess, 38(2), 284-296. doi: 10.1007/ s10862-015-9514-X

Brockmeyer, T., Bents, H., Holtforth, M. G., Pfeiffer, N., Herzog, W., \& Friederich, H. C. (2012). Specific emotion regulation impairments in major depression and anorexia nervosa. Psychiatry Research, 200(23), 550-553. doi: 10.1016/j.psychres.2012.07.009

Brosseau-Liard, P. E., \& Savalei, V. (2014). Adjusting incremental fit indices for nonnormality. Multivariate Behavioral Research, 49(5), 460-470. doi: 10.1080/00273171.2014.933697

Brosseau-Liard, P. E., Savalei, V., \& Li, L. (2012). An investigation of the sample performance of two nonnormality corrections for RMSEA. Multivariate Behavioral Research, 47(6), 904-930. doi: 10.1080/00273171.2012.715252

Brown, T. A. (2015). Confirmatory factor analysis for applied research (2a ed.). New York, NY: Guilford Press. 
Browne, M. W. (1972). Orthogonal rotation to a partially specified target. British Journal of Mathematical and Statistical Psychology, 25(1), 115-120. doi: 10.1111/ j.2044-8317.1972.tb00482.x

Byrne, B. M. (2012). Structural equation modeling with Mplus: Basic concepts, applications, and programming. New York, NY: Routledge.

Campos, J. J., Frankel, C. B., \& Camras, L. (2004). On the nature of emotion regulation. Child Development, 75(2), 377-394. doi: 10.1111/j.1467-8624.2004.00681.x

Caycho-Rodríguez, T., Dominguez-Lara, S., Noe-Grijalva, M., \& Reyes-Bossio, M. (2019). Dimensionalidad de un ítem único de preocupación por el cáncer mediante modelos de ecuaciones estructurales. Revista Argentina de Ciencias del Comportamiento, 11(3), 73-80. doi: 10.32348/1852.4206.v11.n3.23875

Chervonsky, E., \& Hunt, C. (2019). Emotion regulation, mental health, and social wellbeing in a young adolescent sample: A concurrent and longitudinal investigation. Emotion, 19(2), 270-282. doi: 10.1037/ emo0000432

Cole, P. M., Martin, S. E., \& Dennis, T. A. (2004). Emotion regulation as a scientific construct: Methodological challenges and directions for child development research. Child Development, 75(2), 317-333. doi: 10.1111/j.1467-8624.2004.00673.x

Cole, P. M., Michel, M. K., \& Teti, L. O. (1994). The development of emotion regulation and dysregulation: A clinical perspective. Monographs of the Society for Research in Child Development, 59(2-3), 73-102. doi: 10.1111/j.1540-5834.1994.tb01278.x

De France, K., \& Evans, G. W. (2020). Expanding context in the role of emotion regulation in mental health: How socioeconomic status (SES) and developmental stage matter. Emotion. Advance online publication. doi: 10.1037/emo0000743

Dominguez-Lara, S. (2018). Ítem único de ansiedad ante exámenes: Análisis con modelos de ecuaciones estructurales. Enfermería Clínica, 28(2), 143-144. doi: 10.1016/j.enfcli.2017.03.013

Dunn, T. J., Baguley, T., \& Brunsden, V. (2014). From alpha to omega: A practical solution to the pervasive problem of internal consistency estimation. British Journal of Psychology, 105(3), 399-412. doi: 10.1111/bjop.12046

Ferrando, P. J., \& Lorenzo-Seva, U. (2017). Program FACTOR at 10: Origins, development and future directions. Psicothema, 29(2), 236-240. doi: 10.7334/ psicothema2016.304

Flores-Kanter, P., \& Medrano, L. (2018). Comparación de dos versiones reducidas de la Escala PANAS: Análisis factoriales en una muestra argentina. Revista Iberoamericana de Diagnóstico y Evaluación, 49(4), 37-46. doi: 10.21865/RIDEP49.4.03

Flores-Kanter, P. E. (2020). Commentary: How we know what not to think. Frontiers in Psychology, 11. doi: 10.3389/fpsyg.2020.00306

Flores-Kanter, P. E., Dominguez-Lara, S., Trógolo, M. A., $\&$ Medrano, L. A. (2018). Best practices in the use of bifactor models: Conceptual grounds, fit indices and complementary indicators. Revista Evaluar, 18(3), 44-48. doi: 10.35670/1667-4545.v18.n3.22221

Flores-Kanter, P. E., García-Batista, Z. E., Moretti, L. S., \& Medrano, L. A. (2019). Towards an explanatory model of suicidal ideation: The effects of cognitive emotional regulation strategies, affectivity and hopelessness. The Spanish Journal of Psychology, 22, e43. doi: 10.1017/sjp.2019.45

Flores-Kanter, P. E., \& Medrano, L. A. (2020). Commentary: Putting 'Emotional Intelligences' in their place: Introducing the Integrated Model of Affect-Related Individual Differences. Frontiers in Psychology, 11. doi: 10.3389/fpsyg.2020.00574

Garnefski, N., Kraaij, V., \& Spinhoven, P. (2001). Negative life events, cognitive emotion regulation and emotional problems. Personality and Individual Differences, 30(8), 1311-1327. doi: 10.1016/ S0191-8869(00)00113-6

Giromini, L., Velotti, P., de Campora, G., Bonalume, L., \& Cesare-Zavattini, G. (2012). Cultural adaptation of the difficulties in emotion regulation scale: Reliability and validity of an Italian version. Journal 
of Clinical Psychology, 68(9), 989-1007. doi: 10.1002/jclp.21876

Gratz, K. L., \& Gunderson, J. G. (2006). Preliminary data on an acceptance-based emotion regulation group intervention for deliberate self-harm among women with borderline personality disorder. Behavior Therapy, 37(1), 25-35. doi: 10.1016/j.beth.2005.03.002

Gratz, K. L., \& Roemer, L. (2004). Multidimensional assessment of emotion regulation and dysregulation: Development, factor structure, and initial validation of the difficulties in emotion regulation scale. Journal of Psychopathology and Behavioral Assessment, 26(1), 41-54. Recuperado de https://www.springer. com/journal/10862

Gross, J. J., \& John, O. P. (2003). Individual differences in two emotion regulation processes: Implications for affect, relationships, and well-being. Journal of Personality and Social Psychology, 85(2), 348-362. doi: 10.1037/0022-3514.85.2.348

Gross, J. J., \& Levenson, R. W. (1997). Hiding feelings: The acute effects of inhibiting negative and positive emotion. Journal of Abnormal Psychology, 106(1), 95-103. doi: 10.1037/0021-843X.106.1.95

Gross, J. J., \& Thompson, R. A. (2007). Emotion regulation: Conceptual foundations. En J. J. Gross (Ed.), Handbook of Emotion Regulation (pp. 3-26). New York, NY: Guilford Press.

Guzmán-González, M., Trabucco, C., Urzúa, M. A., Garrido, L., \& Leiva, J. (2014). Validez y confiabilidad de la versión adaptada al español de la Escala de Dificultades de Regulación Emocional (DERS-E) en población chilena. Terapia Psicológica, 32(1), 1929. doi: $10.4067 / \mathrm{S} 0718-48082014000100002$

Hallion, L. S., Steinman, S. A., Tolin, D. F., \& Diefenbach, G. J. (2018). Psychometric properties of the Difficulties in Emotion Regulation Scale (DERS) and its short forms in adults with emotional disorders. Frontiers in Psychology, 9. doi: 10.3389/fpsyg.2018.00539

Hernández-Guzmán, L., del Palacio, A., Freyre, M., \& Alcázar-Olán, R. (2011). La perspectiva dimensional de la psicopatología. Revista Mexicana de
Psicología, 28(2), 111-120. Recuperado de http:// comeppsi.com/rmp-28

Hervás, G., \& Jódar, R. (2008). Adaptación al castellano de la Escala de Dificultades en la Regulación Emocional. Clínica y Salud, 19(2), 139-156. Recuperado de https://journals.copmadrid.org/clysa

Hu, L., \& Bentler, P. M. (1999). Cutoff criteria for fit indexes in covariance structure analysis: Conventional criteria versus new alternatives. Structural Equation Modeling: A Multidisciplinary Journal, 6(1), 1-55. doi: 10.1080/10705519909540118

Johnstone, T., \& Walter, H. (2014). The neural basis of emotion dysregulation. En J. J. Gross (Ed.), Handbook of Emotion Regulation (pp. 58-75). New York, NY: The Guilford Press.

Kaufman, E. A., Xia, M., Fosco, G., Yaptangco, M., Skidmore, C. R., \& Crowell, S. E. (2016). The Difficulties in Emotion Regulation Scale short form (DERS-SF): Validation and replication in adolescent and adult samples. Journal of Psychopathology Behavior Assessment, 38(3), 443-455. doi: 10.1007/ s10862-015-9529-3

Kerlinger, F. N., \& Lee, H. B. (2002). Análisis estructural de covarianza. En Investigación del Comportamiento: Métodos de investigación en ciencias sociales (pp. 785-808). México, DF: McGraw Hill.

Kring, A. M., \& Sloan, D. M. (Eds.). (2010). Emotion regulation and psychopathology: A transdiagnostic approach to etiology and treatment. New York, NY: The Guilford Press.

Leahy, R. L., Tirch, D., \& Napolitano, L. A. (2011). Emotion regulation in psychotherapy: A practitioner's guide. New York, NY: Guilford Press.

Lebowitz, M. S., \& Dovidio, J. F. (2015). Implications of emotion regulation strategies for empathic concern, social attitudes, and helping behavior. Emotion, 15(2), 187-194. doi: 10.1037/a0038820

Li, J., Han, Z. R., Gao, M. M., Sun, X., \& Ahemaitijiang, N. (2018). Psychometric properties of the Chinese version of the Difficulties in Emotion Regulation Scale (DERS): Factor structure, reliability, and va- 
lidity. Psychological Assessment, 30(5), e1-e9. doi: 10.1037/pas0000582

Linehan, M. M. (1993). Diagnosis and treatment of mental disorders. Cognitive-behavioral treatment of borderline personality disorder. New York, NY: Guilford Press.

Marín-Tejeda, M., Robles-García, R., González-Forteza, C., \& Andrade-Palos, P. (2012). Propiedades psicométricas de la escala "Dificultades en la Regulación Emocional" en español (DERS-E) para adolescentes mexicanos. Salud Mental, 35(6), 521-526. Recuperado de http://www.revistasaludmental.mx/ index.php/salud_mental

Mattos-Machado, B., Gonçalves-Gurgel, L., GonçalvesBoeckel, M., \& Tozzi-Reppold, C. (2020). Evidences of validity of the Difficulties in Emotion Regulation Scale - DERS. Paidéia, 30. doi: 10.1590/1982-4327e3017

Medrano, L., \& Trógolo, M. (2014). Validación de la Escala de Dificultades en la Regulación Emocional en la población universitaria de Córdoba, Argentina. Universitas Psychologica, 13(4), 15-26. doi: 10.11144/Javeriana.UPSY13-4.vedr

Montero, I., \& León, O. G. (2007). A guide for naming research studies in psychology. International Journal of Clinical and Health Psychology, 7(3), 847-862. Recuperado de https://www.elsevier.es/en-revista-international-journal-of-clinical-and-health-psychology-355

Muñoz-Martínez, A. M., Vargas, R. M., \& Hoyos-González, J. S. (2016). La Escala de Dificultades en Regulación Emocional (DERS): Análisis factorial en una muestra colombiana. Acta Colombiana de Psicología, 19(1), 225-236. doi: 10.14718/ACP.2016.19.1.10

Páez-Rovira, D., Martínez-Sánchez, F., Sevillano-Triguero, V., Mendiburo-Seguel, A., \& Campos, M. (2012). Medida de Estilos de Regulación Afectiva (MARS) ampliada en ira y tristeza. Psicothema, 24(2), 249254. Recuperado de http://www.psicothema.es

Petrescu, M. (2013). Marketing research using single-item indicators in structural equation models. Journal of
Marketing Analytics, 1(2), 99-117. doi: 10.1057/ jma.2013.7

Qu, Y., \& Telzer, E. H. (2017). Cultural differences and similarities in beliefs, practices, and neural mechanisms of emotion regulation. Cultural Diversity and Ethnic Minority Psychology, 23(1), 36-44. doi: 10.1037/ cdp0000112

Reyes-Ortega, M. A., \& Tena-Suck, E. A. (2016). Regulación emocional en la práctica clínica: Una guía para terapeutas. México, DF: Manual Moderno.

Rhemtulla, M., Brosseau-Liard, P. E., \& Savalei, V. (2012). When can categorical variables be treated as continuous? A comparison of robust continuous and categorical SEM estimation methods under suboptimal conditions. Psychological Methods, 17(3), 354-373. doi: $10.1037 / \mathrm{a} 0029315$

Rieffe, C., Oosterveld, P., Miers, A. C., Meerum-Terwogt, M., \& Ly, V. (2008). Emotion awareness and internalising symptoms in children and adolescents: The Emotion Awareness Questionnaire revised. Personality and Individual Differences, 45(8), 756761. doi: 10.1016/j.paid.2008.08.001

Rodriguez, A., Reise, S. P., \& Haviland, M. G. (2016). Evaluating bifactor models: Calculating and interpreting statistical indices. Psychological Methods, 21(2), 137-150. doi: 10.1037/met0000045

Rosseel, Y. (2012). lavaan: An R package for structural equation modeling. Journal of Statistical Software, 48(2), 1-36. doi: 10.18637/jss.v048.i02

Ruganci, R. N., \& Gençöz, T. (2010). Psychometric properties of a Turkish version of the Difficulties in Emotion Regulation Scale. Journal of Clinical Psychology, 66(4), 442-455. doi: 10.1002/jclp.20665

Sauer-Zavala, S., Gutner, C. A., Farchione, T. J., Boettcher, H. T., Bullis, J. R., \& Barlow, D. H. (2017). Current definitions of "transdiagnostic" in treatment development: A search for consensus. Behavior Therapy, 48(1), 128-138. doi: 10.1016/j.beth.2016.09.004

Savalei, V. (2019). A comparison of several approaches for controlling measurement error in small samples. Psychological Methods, 24(3), 352-370. doi: 
10.1037/met0000181

Seligowski, A. V., Lee, D. J., Bardeen, J. R., \& Orcutt, H. K. (2015). Emotion regulation and posttraumatic stress symptoms: A meta-analysis. Cognitive Behaviour Therapy, 44(2), 87-102. doi: 10.1080/16506073.2014.980753

Shahabi, M., Hasani, J., \& Bjureberg, J. (2020). Psychometric properties of the brief Persian version of the Difficulties in Emotion Regulation Scale (The DERS-16). Assessment for Effective Intervention, 45(2), 135-143. doi: 10.1177/1534508418800210

Stewart, I., Villatte, M., \& McHugh, L. (2012). Approaches to the self. En L. McHugh \& I. Stewart (Eds.), The self and perspective taking. Contributions and applications from modern behavioral science (pp. 3-35). Oakland, CA: New Harbinger.

Thompson, R. A. (1994). Emotion regulation: A theme in search of definition. Monographs of the Society for Research in Child Development, 59(2-3), 25-52. doi: 10.1111/j.1540-5834.1994.tb01276.x

Tull, M. T., Bardeen, J. R., DiLillo, D., Messman-Moore, T., \& Gratz, K. L. (2015). A prospective investigation of emotion dysregulation as a moderator of the relation between posttraumatic stress symptoms and substance use severity. Journal of Anxiety Disorders, 29, 52-60. doi: 10.1016/j.janxdis.2014.11.003

Van Beveren, M. L., de Clercq, B., \& Braet, C. (2020). Just the way you are. Understanding emotion regulation strategies in youth from temperamental differences. Journal of Research in Personality, 88, 103989. doi: 10.1016/j.jrp.2020.103989

Vargas-Gutiérrez, R. M., \& Muñoz-Martínez, A. M. (2013). La regulación emocional: Precisiones y avances conceptuales desde la perspectiva conductual. Psicología-USP, 24(2), 225-240. doi: 10.1590/ s0103-65642013000200003

Victor, S. E., \& Klonsky, E. D. (2016). Validation of a brief version of the Difficulties in Emotion Regulation Scale (DERS-18) in five samples. Journal of Psychopathology Behavior Assessment, 38(4), 582589. doi: 10.1007/s10862-016-9547-9
Weinberg, A., \& Klonsky, E. D. (2009). Measurement of emotion dysregulation in adolescents. Psychological Assessment, 21(4), 616-621. doi: 10.1037/a0016669

Weiss, N. H., Gratz, K. L., \& Lavender, J. M. (2015). Factor structure and initial validation of a multidimensional measure of difficulties in the regulation of positive emotions: The DERS-Positive. Behavior Modification, 39(3), 431-453. doi: 10.1177/0145445514566504

Wong, C. F., Silva, K., Kecojevic, A., Schrager, S. M., Bloom, J. J., Iverson, E., \& Lankenau, S. E. (2013). Coping and emotion regulation profiles as predictors of nonmedical prescription drug and illicit drug use among high-risk young adults. Drug and Alcohol Dependance, 132(1-2), 165-171. doi: 10.1016/j. drugalcdep.2013.01.024

Yiğit, I., \& Guzey-Yiğit, M. (2019). Psychometric properties of Turkish version of Difficulties in Emotion Regulation Scale-Brief Form (DERS-16). Current Psychology, 38(6), 1503-1511. doi: 10.1007/s12144017-9712-7

Yuan, K.-H., \& Bentler, P. M. (2000). Three likelihood-based methods for mean and covariance structure analysis with nonnormal missing data. Sociological Methodology, 30(1), 165-200. doi: 10.1111/0081-1750.00078 